INTERNACIONAL

\title{
La construcción del estigma como límite a los derechos sociales de las personas trans desde una perspectiva internacional
}

\author{
Stigma construction as a boundary to transgender people's social rights \\ from an international approach
}

Eduardo Arrubia y Mariana Brocca

Universidad Nacional del Centro de la Provincia de Buenos Aires, Argentina

\begin{abstract}
RESUMEN La igualdad formal constituye una reivindicación necesaria para lograr el reconocimiento estatal de las personas LGBTI. Sin embargo, puede advertirse que más allá de las normas jurídicas existe una plataforma fáctica de desigualdad en la que se construye el estigma, al punto de impedir el goce efectivo de derechos sociales como la educación y la salud, entre otros. En este orden, los Principios de Yogyakarta sobre la aplicación de la legislación internacional de derechos humanos en relación con la orientación sexual y la identidad de género ilustran las expectativas de satisfacción de derechos de este colectivo. Es por ello que puede advertirse respecto a personas trans, a través de casos y documentos oficiales que han surgido en el último año en distintos países, que estos principios han cobrado mayor virtualidad. Así, el material analizado en este trabajo refleja que el estigma contra la diversidad sexual se construye en el ámbito más inmediato de la cotidianidad, lo cual exige que el respeto hacia los derechos de este grupo debe comenzar en los niveles próximos de los derechos domésticos por medio de políticas públicas con enfoque de derechos.
\end{abstract}

PALABRAS CLAVE Personas trans, LGBTI, Discriminación, DESC.

ABSTRACT Formal equality constitutes a paramount vindication in order to achieve the state acknowledgment of LGBTI people. However, it can be observed that there is a factual platform of inequality beyond juridical norms, whereby stigma is constructed so as to impede the effective enjoyment of social rights such as education and health, among others. In this sense, the Yogyakarta Principles on the Application of International Human Rights Law in relation to Sexual Orientation and Gender Identity illustrate the expectations of compliance with this group's rights. Therefore, it can be noticed 


\begin{abstract}
that these principles have attained major fulfilment throughout cases and official papers, which have emerged in different countries over last year. Then, the material analysed in this essay reflects that the stigma against sexual diversity is constructed in the immediate scope of daily life. This demands that the respect towards LGBTI people's rights shall begin from the closest levels of domestic laws based on public policies designed with a human rights approach.
\end{abstract}

KEYWORDS Transgender people, LGBTI, discrimination, ESCR.

\title{
Introducción
}

La indagación acerca de los derechos sociales de las personas trans supone de antemano la afirmación lógica de que existe otra categoría de derechos humanos que no serían «sociales». Al respecto, debemos dejar en claro que si bien no compartimos la clasificación generacional de los derechos, menos aún cuando es utilizada para negar la exigibilidad de los llamados derechos económicos, sociales y culturales (DESC), reconocemos que dicha rotulación tiene cierto valor heurístico, es decir, explicativo (Abramovich y Courtis, 2002: 27), que adquiere utilidad a los efectos de individualizar y narrar las experiencias de vulnerabilidad de este colectivo. En este sentido, la Comisión Interamericana de Derechos Humanos (CIDH) ha advertido que las personas LGBTI y en especial las personas trans enfrentan un ciclo de exclusión y pobreza que profundiza la exposición cotidiana a situaciones de violencia $(\mathrm{CIDH}$, 2015: 217). En un esquema en que la igualdad formal en términos de derechos civiles (por ejemplo, derecho al matrimonio, a la filiación, a la identidad de género, a la vida privada, etcétera) aparece como una reivindicación necesaria para lograr el reconocimiento estatal, la heterogeneidad de las vivencias particulares evidencia que la desigualdad material atraviesa a este colectivo en cuanto a la salud y la educación, entre otros de los derechos económicos, sociales y culturales. Existe una heteronormatividad cultural que determina y disciplina a las personas al punto de definir el goce efectivo de derechos o la privación de ellos.

En este orden de ideas, la Corte Interamericana de Derechos Humanos (Corte IDH) ha sostenido que a la hora de interpretar el sentido de la expresión «otra condición social», propia del artículo 1.1 de la Convención Americana sobre Derechos Humanos $(\mathrm{CADH})$, corresponde escoger la alternativa hermenéutica más favorable a la tutela de los derechos de la persona humana, conforme a la aplicación del principio pro persona ${ }^{1}$ Por eso, en la misma ocasión este tribunal internacional ha reconocido que los derechos relativos a la diversidad sexual están amparados por la norma cita-

1. Sentencia de fondo, reparaciones y costas del caso Atala Riffo y niñas contra Chile, Corte IDH, Serie C núm. 239, 24 de febrero de 2012, foja 84 . 
$\mathrm{da}^{2}$. Para esto, se ha hecho expresa referencia a las resoluciones que la Asamblea General de la Organización de los Estados Americanos ha adoptado, como así también a la jurisprudencia del Tribunal Europeo de Derechos Humanos que en el mismo sentido ha incluido a la orientación sexual en el marco normativo del artículo 14 del Convenio Europeo de Derechos Humanos. Con idéntico alcance ha sido interpretada la expresión análoga contenida en el artículo 2 de la Convención Internacional sobre los Derechos del Niño, dentro de la cual el Comité de control de este instrumento ha establecido que la orientación sexual se encuentre vedada como motivo de discriminación (Comité de los Derechos del Niño, 2003: párrafo 6).

Entonces, tal como ha quedado dicho, estos despliegues del derecho internacional evidencian avances trascendentes en cuanto a la tutela del colectivo de la diversidad sexual. Sin embargo, surge el interrogante de cuál es el estado de situación de los derechos sociales de las minorías sexuales más allá de estos desarrollos. Para analizar el problema, nos limitamos al análisis del derecho a la educación y del derecho a la salud de personas trans, sin pretender agotar con ellos el universo de los derechos económicos, sociales y culturales. Tomamos el universo de personas trans porque consideramos que es prudente desplazar la mirada del reconocimiento anclado en la identidad en términos de pertenencia al grupo LGBTI, para pensarlo en clave de estatus individual de cada miembro del colectivo en tanto participante de la interacción social (Fraser, 2000: 61). Con esta finalidad, abordaremos casos judiciales que han trascendido en el último año en países de Latinoamérica, los que sumados a otros documentos oficiales en la materia vienen demostrando la operatividad de los Principios de Yogyakarta sobre la aplicación de la legislación internacional de derechos humanos en relación con la orientación sexual y la identidad de género. De esta manera, asumimos el objetivo de dar visibilidad a la problemática trans mediante el análisis de la jurisprudencia aquí citada.

\section{La identidad de género en la educación}

En relación con el derecho a la educación, debe reconocerse ante todo que éste constituye un multiplicador, es decir, su goce efectivo aumenta el disfrute de todos los derechos y libertades en general (Tomasevski, 2003: 11). El proceso educativo aparece regulado desde la normativa internacional no sólo como mero derecho, sino también en relación con el rol que los derechos humanos conllevan en la educación y en su vinculación como contenido de la enseñanza (Tomasevski, 2003: 12). Por lo tanto, en el marco del paradigma de los derechos humanos, la educación ya no puede fundamentarse únicamente en una racionalidad técnica que verse sobre parámetros de cientificidad, sino que además debe necesariamente incorporar un enfoque de

2. Atala Riffo y niñas contra Chile, foja 91. 
racionalidad práctica (Hevia, 2006: 73). Esto implica pensar la educación no sólo en términos de contenidos duros, sino a través de los valores inherentes a la humanidad misma, a la aceptación y el respeto por el prójimo. En este sentido, los Principios de Yogyakarta antedichos resultan ilustrativos, ya que establecen la protección de la igualdad de condiciones en cuanto al acceso de los estudiantes al sistema educativo, pero también el trato igualitario en clave de orientación sexual e identidad de género no sólo de éstos, sino también de los docentes y el personal en general's.

En este orden, advertimos que el escenario jurídico latinoamericano viene dando señales que adjudican virtualidad a estos principios. Así, en 2016 dos sentencias valiosas en esta materia fueron dictadas por dos máximos tribunales, esto es, la Corte Constitucional de Colombia y la Suprema Corte de Justicia de México. El primero se trata de un caso vinculado a un hombre trans, es decir, una persona que biológicamente nació como mujer pero que siente su género como hombre. Por lo tanto, en consonancia con dicho sentir peticionó al Servicio Nacional de Aprendizaje colombiano (SENA) que le fuera permitido usar en la institución educativa un uniforme masculino acorde con su género autopercibido y que se le dispensara un trato igualitario en las aulas de clases por parte del cuerpo de docentes, que se refirieran a su persona con sustantivos y artículo masculinos y que se abstuviesen de atentar de manera alguna contra su personalidad y forma de ser ${ }^{4}$. Frente a la negativa de respuesta por parte de la entidad, se inició una acción de tutela. En esta oportunidad, los argumentos esgrimidos por el SENA se concentraron en exigir al estudiante que modificara la inscripción registral de su género y, por consiguiente, su documento de identidad. Así, se buscó condicionar el ejercicio del derecho a un trato digno supeditando algo tan simple como la vestimenta de un uniforme a un trámite registral de reasignación de sexo. La acción de tutela fue concedida por un juez de segunda instancia y luego la Corte Constitucional colombiana determinó que el SENA había vulnerado los derechos a la igualdad y al libre desarrollo de la personalidad del estudiante, al haberse negado a brindarle un trato acorde con su identidad de género como hombre transexual y al haberle prohibido utilizar el uniforme atribuido a los aprendices de género masculino y lucir el cabello corto, bajo el argumento de que la información que el accionante había suministrado al momento de su inscripción correspondía al sexo femeninos.

Por otra parte, como hemos señalado, la relación entre educación y derechos humanos debe ser abordada desde una mirada multidimensional que permita ahondar

3. Principios de Yogyakarta sobre la aplicación de la legislación internacional de derechos humanos en relación con la orientación sexual y la identidad de género, principio 16. Disponible en http://bit. ly/2iBpGov.

4. Sentencia de la Corte Constitucional colombiana, T-363/16, 11 de julio de 2016, foja 19, apartado 2.2.

5. Corte Constitucional colombiana, apartado 2.37. 
en todas las implicancias que pueda tener el derecho a la educación. En este sentido, el pronunciamiento del Superior Tribunal mexicano constituye una pieza clave al consolidar el acceso a una formación educativa en materia de diversidad sexual desde la infancia como una herramienta esencial para el desarrollo potencial y pleno de la personalidad humana. En esta ocasión, la Corte mexicana se expidió en un caso en el cual la madre de un niño alegó la presunta inconstitucionalidad de la Ley General de los Derechos de los Niños, Niñas y Adolescentes, ya que esta norma contempla la educación sexual refiriéndose a la "preferencia sexual», como así también al uso de métodos anticonceptivos ${ }^{6}$. Según su parecer, la accionante consideraba que, de este modo, el Estado se entromete ilegítimamente en sus derechos inherentes a la patria potestad. Al respecto, la Corte sostuvo que precisamente el artículo 1 de la Constitución de México asume el mandato de prohibición de discriminación por los motivos que allí se enuncian, entre los cuales destaca expresamente el de «preferencia sexual»?. Por consiguiente, resulta que la norma que la accionante intenta impugnar es consecuente con la Ley Fundamental del Estado mexicano, sobre todo si se considera que la finalidad de la misma está enmarcada en el resguardo del interés superior del niño, de manera congruente con la tutela que emana de la Convención Internacional sobre los Derechos del Niño, y que ella de ninguna forma está encaminada a determinar la sexualidad de los infantes, sino que, por el contrario, su misión radica en inculcar el respeto a la diversidad sexual evidenciando la pluralidad de preferencias sexuales que pueden asumirse ${ }^{8}$. En esta línea, se derrumba la alusión a la incolumidad de la patria potestad, ya que se entiende que de ninguna manera puede anular o sustituir la voluntad de un niño, en este caso vinculada a la elección de la sexualidad sentida9 ${ }^{9}$.

En relación con esta última sentencia, advertimos cierta vaguedad en la Constitución mexicana, que sólo contempla como categorías prohibidas de discriminación al «género» y a las «preferencias sexuales». Sin embargo, sostenemos que, aunque no es lo mismo hablar de identidad de género que de preferencias sexuales, aquella aparece incluida dentro de éstas. De este modo, la identidad de género queda comprendida como una expresión más de la sexualidad humana, digna de ser respetada por la sociedad y abarcada desde la planificación educativa que está destinada a engendrar dicho respeto en los infantes. Simultáneamente, advertimos que estos pronunciamientos evidencian el cumplimiento de los estándares que el comité de DESC ha fijado para el derecho a la educación (CESCR, 1999: párrafo 6). Así, se logra la accesibilidad, ya que se reprocha una práctica discriminatoria que impedía a un estudiante asumir

6. Sentencia de la Suprema Corte de Justicia de México, amparo de revisión 203/2016, 9 de noviembre de 2016, fojas 13-14.

7. Suprema Corte de Justicia de México, foja 17.

8. Suprema Corte de Justicia de México, foja 18.

9. Suprema Corte de Justicia de México, foja 53. 
externamente su propia identidad de género en el establecimiento educativo. En el caso mexicano, la aceptabilidad exigida para este derecho es lograda a través de la reivindicación de la constitucionalidad de una norma que establece contenidos educativos en materia de respeto por la diversidad sexual. La característica de la adaptabilidad está presente en ambas decisiones, ya que se refuerzan prácticas educativas acordes con los nuevos tiempos en que los derechos humanos exigen el respeto a la diversidad sexual.

En definitiva, el panorama internacional reciente que podemos ejemplificar con los dos casos jurisprudenciales aquí reseñados demuestra en el terreno fáctico la existencia de prejuicios y estigmas que amenazan el pleno goce de los derechos humanos referidos a la sexualidad en el plano educativo. Esto se ve reflejado en hechos tales como el de un estudiante a quien no se le dispensa un trato digno de parte de autoridades escolares y docentes, ya que amedrentan su derecho personalísimo a la identidad de género, con lo que menoscaban su personalidad en virtud de un documento formal en el que consta su género. Asimismo, la preocupación de una madre que llega al punto de accionar judicialmente para impugnar una norma estatal, en el entendido de que es perjudicial para su hijo que sea formado en asuntos de sexualidad, pone en evidencia las tensiones que socialmente experimentan los derechos del colectivo LGBTI. En este contexto de conflicto, la actividad judicial ha operado favorablemente en aras de lograr el respeto y el desarrollo de las prerrogativas fundamentales de la diversidad sexual que redundan en beneficio propio y de la sociedad en general. Además, este escenario muestra la necesidad de desarrollar políticas públicas con enfoque de derechos, es decir, que garanticen los estándares internacionales más elementales en materia de derechos humanos. Éstos se basan en la obligación de satisfacer, por lo menos, el contenido mínimo de los derechos y de utilizar al máximo los recursos disponibles, en el principio de progresividad y no regresividad, en el principio de no discriminación, en la producción de información, en la participación de los sectores afectados en el diseño de las políticas públicas y en el acceso a la justicia (Abramovich y Pautassi, 2009: 283).

\section{Bienestar y diversidad sexual: el derecho a la salud como elemento constitutivo de la identidad de género}

En consonancia con el argumento que venimos desarrollando, enfocado en la desigualdad material más allá de las normas formales, puede afirmarse que a pesar de que el derecho a la salud se encuentre regulado en todos aquellos tratados dedicados a los derechos económicos, sociales y culturales, lo cierto es que la comunidad LGBTI ha debido tolerar continuos detrimentos a este derecho (ACNUDH, 2011: párrafos 54-57). En este sentido, surge a partir de trabajos doctrinarios que, dentro del ámbito público, el sector sanitario es el que más discrimina negativamente a las personas por 
su orientación sexual e identidad de género (Jaime, 2013: 17; Godoy, González y Rivero, 2013: 10-17). En efecto, se ha logrado constatar la existencia de prácticas discriminatorias generalizadas por parte de los profesionales de la salud hacia la comunidad LGBTI en los centros sanitarios (CIDH, 2015: párrafo 196). A modo de ejemplo, el maltrato, la falta de confidencialidad y el retraso injustificado en la atención son algunos de los hechos más comunes (ACNUDH, 2011: párrafo 56).

Conforme lo ha podido constatar la CIDH, las personas LGBTI «han visto su salud deteriorada rápidamente a causa de la negativa de asistencia médica, e incluso casos de muertes que pudieron ser prevenidas si se hubiese proporcionado un tratamiento médico apropiado» (CIDH, 2015: párrafo 199). Asimismo, la violencia y los prejuicios contra la comunidad LGBTI han ocasionado que gran parte de ella se abstenga de asistir a dichos establecimientos, lo que trae como consecuencia que su salud alcance un estado sumamente crítico (CIDH, 2015: párrafos 198-199). Así:

La Oficina Panamericana de la Salud (OPS) y la Organización Mundial de la Salud (OMS) han identificado en algunas de sus publicaciones que la homofobia, la discriminación y el estigma hacia las personas por su orientación sexual e identidad sexual y de género constituye una barrera para el acceso de las personas a los servicios de salud, a la consulta y la continuación de tratamientos (Godoy, González y Rivero, 2013).

En esta línea de pensamiento, ciertos países de Latinoamérica cuentan con informes recientes en el tema. En efecto, la Defensoría del Pueblo de Perú, a través de su Informe de 2016, ha podido observar que:

Los prejuicios y estereotipos hacia las personas LGBTI inciden en el tipo de tratamiento brindado por los profesionales de salud. De los testimonios recabados e informes publicados por sus organizaciones, se desprende que cuando una persona de este colectivo se acerca a un establecimiento de salud por una dolencia cualquiera y es identificada como tal, una reacción común que obtiene del personal de salud es interrogarla sobre si tiene alguna infección de transmisión sexual (ITS) o viven con el VIH (Defensoría del Pueblo, 2016: 145).

Asimismo, la Fundación Ecuatoriana Equidad, en su último informe en el tema, presentado en mayo de 2016, ha expuesto que:

Entre las problemáticas graves que se evidenciaron en Ecuador se encuentran: inexistente acceso a tratamiento hormonal y otras terapias para personas trans a través de agencias estatales de salud, la falta de educación y prevención de VIH en personas trans (la educación se centra en hombres gays) y un ineficiente sistema de salud estatal que no cuenta con estudios y estadísticas propias sobre la prevalencia de VIH en la comunidad LGBTI, ni métodos de prevención y tratamiento con protocolos que tengan enfoque especializado. A todo esto se le debe sumar el estigma 
y discriminación que sufre la población gay y trans con respecto al VIH y la irresponsabilidad del estado ecuatoriano con respecto a la prevención de esta epidemia (Fundación Ecuatoriana Equidad, 2016: 7-8).

En relación con esto, debe dejarse constancia de que los Principios de Yogyakarta antes mencionados incluyen el derecho a la salud, que establece el derecho al disfrute del más alto nivel posible de salud y la protección contra abusos médicos.

En el caso de Argentina, en donde el derecho a la identidad de género aparece reconocido por una ley especial, en el año 2015 el Poder Ejecutivo reglamentó la incorporación de determinadas prácticas médicas al Plan Médico Obligatorio. Esto implica que dichos tratamientos son de cobertura obligatoria para las obras sociales y constituyen prestaciones que el Estado se obliga a otorgar de manera gratuita. Al respecto, es importante traer a colación un caso de la jurisprudencia argentina que fue resuelto en la ciudad de Rosario ${ }^{10}$. En esa oportunidad, una mujer trans demandó a su obra social por negarse a dar cobertura a los tratamientos de gluteoplastía de aumento y depilación definitiva de rostro. La parte demandada alegó en su defensa que se trataba de meros tratamientos estéticos que en nada se relacionaban con la salud de la afiliada. Sin embargo, resulta muy valioso el pronunciamiento del juez que dictaminó que cuando estas prácticas están vinculadas a la asunción de una corporalidad diferente del género atribuido culturalmente a la persona, lo que está en juego es la identidad misma del sujeto y no una finalidad meramente estética. Así, estos tratamientos se imponen como indispensables para resguardar el derecho a la salud que va ligado a la identidad de género de la persona, ya que en casos como éstos forman parte del completo bienestar físico, emocional y social, que redunda intrínsecamente en la identidad personal.

\section{A modo de cierre}

En suma, entendemos que el avance más sistematizado de protección a los derechos de la comunidad LGBTI en el plano internacional se encuentra reflejado en los Principios de Yogyakarta antes mencionados. A pesar de no ser un instrumento convencional vinculante, su formulación deviene de imperiosa utilidad como pauta hermenéutica para iluminar la comprensión de la vulnerabilidad que esta población experimenta. Asimismo, debemos destacar que dicha experiencia de debilidad jurídica queda evidenciada en el marco fáctico, que plaga a la diversidad sexual con prejuicios y estereotipos que atentan directamente contra el disfrute de los derechos más elementales de la persona humana. La igualdad formal constituye siempre un primer paso necesario para el reconocimiento del sujeto de derecho en cuanto tal. Pero dicho

10. Sentencia de Juzgado Civil y Comercial, número 6 SS con IAPOS, del 21 de septiembre de 2016. 
reconocimiento se desvanece si no es acompañado de una cultura de respeto en las vivencias más inmediatas de la cotidianidad. De esta manera, los casos aquí analizados muestran un esquema material de acumulación de desventajas socialmente condicionadas en la vida de las personas LGBTI.

Las sentencias de los organismos judiciales tienen un enorme valor ejemplarizante. Sin embargo, es sumamente necesario arribar al entendimiento de que estos niveles de estigma sólo podrán ser erradicados en el marco de una cultura democrática en la que los distintos niveles y poderes del Estado se articulen para configurar políticas públicas con un enfoque de derechos. Sólo de esta manera podrá apuntarse a un plano más profundo que el mero reconocimiento, es decir, un plano de construcción de una conciencia moral de la humanidad acerca de los derechos humanos y de la aberración inherente a toda acción dirigida a desconocerlos (Nino, 2007: 4).

\section{Referencias}

Abramovich, Víctor y Christian Courtis (2002). Los derechos sociales como derechos exigibles. Madrid: Trotta.

Abramovich, Víctor y Laura Pautassi (2009). «El enfoque de derechos y la institucionalidad de las políticas sociales». En Víctor Abramovich y Laura Pautassi (editores), La revisión judicial de las políticas sociales. Estudio de casos (pp. 279-340). Buenos Aires: Editores del Puerto.

ACNUDH, Alto Comisionado de las Naciones Unidas para los Derechos Humanos (2011). «Leyes y prácticas discriminatorias y actos de violencia cometidos contra personas por su orientación sexual e identidad de género». A/HRC/19/41. Disponible en http://bit.ly/2uFf9Ph.

$\mathrm{CIDH}$, Comisión Interamericana de Derecho Humanos (2015). «Violencia contra personas lesbianas, gais, bisexuales, trans e intersex en América». OAS/Ser.L/V/ II.rev.2. Disponible en http://bit.ly/2vyAKoV.

CESCR, Comité de Derechos Económicos, Sociales y Culturales (1999). «Observación General número 13». E/C.12/1999/10. Disponible en http://bit.ly/2uzosz2.

Comité de los Derechos Del Niño (2003). «Observación General número 4. La salud y el desarrollo de los adolescentes en el contexto de la Convención sobre los Derechos del Niño». CRC/GC/2003/4. Disponible en http://bit.ly/2u4XZY3.

Defensoría del Pueblo (2016). Derechos humanos de las personas LGBTI. Necesidad de una política pública para la igualdad en el Perú. Lima.

Fraser, Nancy (2000). "Nuevas reflexiones sobre el reconocimiento». New Left Review, 4: 55-68. Disponible en http://newleftreview.es/4.

Fundación ECUATORIANA EQUIDAD (2016). Resumen del informe en curso sobre la situación de los derechos humanos de las poblaciones LGTBI. Quito.

Godoy, Diana, Soraya GonzÁlez y Javier Rivero (2013). «Componentes de forma- 
ción para la atención en salud de personas sexo-género diversas», Comunidad y Salud, 11 (1): 10-17. Disponible en http://bit.ly/2tCJwkl.

Hevia, Ricardo (2006). «Frente a la crisis de sentido, una pedagogía de la confianza». Revista PRELAC, 2: 70-75. Disponible en http://bit.ly/2tvon9x.: http://unesdoc. unesco.org/images/o014/o01455/145502s.pdf\#nameddest $=145879$.

JAIME, Martín (2013). Diversidad sexual, discriminación y pobreza frente al acceso a la salud pública. Demandas de la comunidad TLGBI en Bolivia, Colombia, Ecuador y Perú. Buenos Aires: CLACSO.

Nino, Carlos (2007). Ética y derechos humanos. Buenos Aires: Astrea.

Tomasevski, Katarina (2001). Contenido y vigencia del derecho a la educación. Serie Cuadernos pedagógicos núm. 6. San José: IIDH. Disponible en http://bit. ly/2gXs6hb.

\section{Sobre los autores}

Eduardo Arrubia es abogado. Becario de investigación doctoral del Consejo Nacional de Investigaciones Científicas y Técnicas de la República Argentina (Conicet). Docente auxiliar interino en la cátedra de Filosofía del Derecho y Ética de la Abogacía, Facultad de Derecho, Universidad Nacional del Centro de la Provincia de Buenos Aires (Unicen). Doctorando en Derecho, UBA. Miembro del Centro de Estudios en Derechos Humanos (CEDH-Unicen). Integrante del Foro Ciudadano de Participación por la Justicia y los Derechos Humanos (FOCO). Su correo electrónico es eduardo.arrubia@azul.der.unicen.edu.ar.

Mariana Brocca es estudiante avanzada de la carrera de Abogacía de la Facultad de Derecho, Unicen. Auxiliar alumna de la cátedra de Derechos Humanos y Garantías de dicha carrera. Becaria de investigación del Consejo Nacional Interuniversitario de la República Argentina. Miembro del Centro de Estudios en Derechos Humanos (CEDH-Unicen). Su correo electrónico es mariana.brocca@azul.der.unicen.edu.ar. 